\title{
Planning of the educational and training process taking into account the accelerated psychophysiological recovery of athletes using digital technologies
}

\author{
Vitaly Belmach ${ }^{1}$, Svetlana Grigan ${ }^{2}$, and Alla Vishina $^{2}$ \\ ${ }^{1}$ Don State Technical University, 344000, Rostov-on-Don, Russia \\ ${ }^{2}$ Rostov State Transport University, 344038, Rostov-on-Don, Russia
}

\begin{abstract}
The article shows that new technologies radically change education and the training process in general. In particular, the context of physical education and the training process also faces some important questions regarding why, when and whether it is necessary to include digital technologies in the training process. Pedagogical knowledge is merged in various ways with technological and substantive knowledge; for this integration of different types of knowledge, it is necessary to develop a new "digital platform". A high level of physical activity during training entails the use of the maximum capabilities of the human body. Therefore, it is necessary to have a clear program in which the rhythm of training is traced by days, weeks and months, the obligatory alternation of load and rest is observed. The aim of the study is to identify the links between physical work and psychophysiological recovery, as well as to substantiate the need to use digital technologies to ensure the effectiveness of the training process.
\end{abstract}

\section{Introduction}

New technologies are fundamentally changing education and the training process in general. In particular, the context of physical education and the training process also faces some important questions regarding why, when and whether it is necessary to include digital technologies in the training process. In sports science, the development of information technologies is reflected in the development of automated methods of functional diagnostics, short-term and long-term adaptation of the body, expert systems, and provides an assessment of the functional readiness of athletes, modeling, forecasting, designing individual components of the sports training system, as well as assessing the effectiveness of the training process. There is now a tendency to assume that today's athletes differ from those of previous generations in the way they learn and train using digital technology. This idea is so deeply rooted that many researchers and trainers regard it as a self-evident truth $[1,2,3,4]$.

\footnotetext{
${ }^{*}$ Corresponding author: (svetlana-grigan@mail.ru)
} 
We use the term "digital technology" to refer to a wide variety of tools, devices, programs and resources that store and transmit information in digital format, such as computers, the Internet, email, mobile phones and other mobile devices, cameras, video games, and what became known as Web 2.0 technologies (e.g., blogs, and social media) [5, $6,7,8]$.

Several names coined for this generation highlight their affinity and propensity to use digital technology. These include millennials, networked generation, digital natives, digital learners, and learners of the digital era $[9,10,11,12]$.

Indeed, some of these studies show that athletes and coaches of the same age differ greatly in how they use technology. In addition, the use of digital technologies does not necessarily entail a great deal of knowledge about them, such as knowledge of programming languages. The technology used by athletes and coaches does not necessarily imply that they use it in their athletic and coaching activities [12, 13, 14].

Athletes and coaches have a certain degree of proficiency in the use of digital technologies, their frequency of use varies depending on the purpose or the specific task for which they are using them. For most athletes, the technological environment is obviously seen as a means of socialization and communication (personal, social and training), in which digital technologies play an important role. Athletes and coaches see cyberspace as an opportunity to meet their communication needs. These results also show that information technology is the most important application for athletes and coaches, as it allows them to connect with each other, obtain information about the athlete's condition over long distances, and stay in touch with coaches sharing a common interest, regardless of their physical location.

This gives us an initial understanding of how athletes and coaches are using technology for training and social purposes, and how these uses are related. This can be very helpful in identifying a specific educational intervention of an initiative and as a point of reflection on the tools that athletes have fully incorporated into their lives. Finally, it is necessary to encourage research and the introduction of experience in information technology, which is consciously applied in the training process. This could lead to a rethinking of the training model and methodology associated with that model.

Finally, the results of this study highlight the benefits of technologies:

a) for collaboration, given their potential for use almost anywhere, anytime;

b) their important role in learning and through the integration of functions, which offers educational, social and technological potential in the training process.

A high level of physical activity during training entails the use of the maximum capabilities of the human body. Therefore, it is necessary to have a clear program in which the rhythm of training is traced by days, weeks and months, the obligatory alternation of load and rest is observed.

The importance of these principles increases with increasing training loads. If a few years ago swimmers who swam more than $3 \mathrm{~km}$ per day were considered an exception, now the load has increased 10-20 times.

A direct relationship between the total mileage and the growth of sports results has been proven. In addition, swimming speed increased during training. Considering the time required for the recovery process, it is theoretically impossible to assume that the athlete will have time to complete all the tasks in the allotted time frame.

From the training theory of previous years, it was revealed that the athlete's body adapts to the increase in physical activity in the period preceding the next training session. The theory, however, has not been confirmed in recent years. Despite the general physical fatigue, you can train and show good results. It is imperative to control the recovery process after training and competition loads and a holistic approach to training and recovery. If the holistic nature of the training process and recovery is not taken into account, a decrease in 
athletic performance is often observed, as well as painful disorders of various body functions.

The aim of the study is to identify the links between physical work and psychophysiological recovery, describe the methods of psychophysiological recovery used after training and competitive loads, as well as substantiate the need to use digital technologies for the effectiveness of the training process.

\section{Materials and methods}

The research base was made up of electronic educational platforms and publications, mobile applications. We studied 1000 anonymous reviews (360 athletes-swimmers) regarding the mobility and the effectiveness of the use of digital technologies in the training process. Moreover, anonymous questioning of the coaching staff was conducted.

The analysis of the literature [1 - 15] on the problems of using electronic devices in the training process in swimming and the methods of psychophysiological recovery used by athletes after training and competitive loads has been carried out.

After analyzing the literature and reviews, an information program was selected that automated the accounting system of planning and control of athletes, and which allows the calculation and analytical way to construct models of various options for building training, taking into account the methods of psychophysiological recovery used after training and competitive loads, to compare them and select the preferred ones, taking into account the athlete's psychophysiological recovery, thereby overcoming, to a certain extent, the limited sampling of empirical material with which coaches are dealing while planning a real educational and training process.

\section{Results}

The experiment involved 20 athletes of qualification 1 adult category and Candidates of Mater of Sports (CMS), divided into two groups - control and experimental (focus), 10 girls in each. The age of the participants is 13-15 years old. The duration of the experiment was 8 weeks of specialized training. When preparing athletes of the experimental group, digital platforms were used that controlled the current state of the athlete, recovery after training and, based on the analysis, determined adjustments to the training process. In the control group, the lessons were conducted according to the generally accepted methodology.

According to the results of the pedagogical experiment, the analysis of changes in the level of preparedness of the swimmers of the control and experimental groups was carried out by assessing the values of the increase in swimming speed, taking into account the average swimming speeds shown by the athletes in the previous tests and tests shown after the experiment.

We do not train today like we did 15 years ago, and in fifteen years we will not train like we do today. Monitoring has changed dramatically, and the evolution of technology and knowledge requires us to move forward and apply technical progress in the training process.

Professionals should have the knowledge and understanding of how to choose an up-todate and effective technology for their work, how to improve the interaction between a coach and an athlete, and how, with the help of technology, to accelerate the operational control over the recovery of the psychological state of an athlete. Digital technologies can help in objectifying the training process, take into account the psychophysiological state of an athlete, and allow more efficient planning and management of the training process. 
Table 1. Dynamics of the level of preparedness of swimmers of the testing and experimental groups during the pedagogical experiment

\begin{tabular}{|c|c|c|c|c|c|c|c|}
\hline \multirow{3}{*}{$\begin{array}{l}\text { Sports } \\
\text { distance }\end{array}$} & \multirow{3}{*}{$\begin{array}{l}\text { Measured } \\
\text { parameter }\end{array}$} & \multicolumn{6}{|c|}{ rate of change in indicator } \\
\hline & & \multicolumn{2}{|c|}{$\begin{array}{l}\text { in the control } \\
\text { group }\end{array}$} & \multicolumn{2}{|c|}{$\begin{array}{l}\text { in the experimental } \\
\text { (focus) group }\end{array}$} & \multicolumn{2}{|c|}{$\begin{array}{c}\text { difference } \\
\text { between } \\
\text { performance of } \\
\text { the groups }\end{array}$} \\
\hline & & value & $\%$ & value & $\%$ & value & $\%$ \\
\hline \multirow{2}{*}{$50 \mathrm{~m}$} & $\mathrm{v}, \mathrm{m} / \mathrm{sec}$ & 0.012 & \multirow[t]{2}{*}{0.6} & 0.021 & \multirow[t]{2}{*}{1.1} & 0.009 & \multirow[t]{2}{*}{0.5} \\
\hline & $\mathrm{t}, \mathrm{m} / \mathrm{sec}$ & 0.17 & & 0.29 & & 0.12 & \\
\hline \multirow{2}{*}{$100 \mathrm{~m}$} & $\mathrm{v}, \mathrm{m} / \mathrm{sec}$ & 0.03 & \multirow[t]{2}{*}{1.8} & 0.062 & \multirow[t]{2}{*}{3.7} & 0.032 & \multirow[t]{2}{*}{1.9} \\
\hline & $\mathrm{t}, \mathrm{m} / \mathrm{sec}$ & 1.07 & & 2.16 & & 1.09 & \\
\hline
\end{tabular}

In recent times, it has become apparent that nutrition, lifestyle, physiotherapy, etc. are of great importance in the daily life of athletes. N. D. Graevskaya, L. A. Ioffe (1973) made a significant contribution to the study of the process of psychophysiological recovery [15]. In the early 2000s, more and more publications began to appear on psychophysiological recovery, most of which dealt with theoretical issues, as well as descriptions of various individual methods.

There have been no studies on how to incorporate psychophysiological recovery into the training process. Usually this problem is solved at the discretion of the trainer, doctor. While some methods of accelerated psychophysiological recovery after training and competitive loads, as well as the technology of their application in practice, have become well-known, the question of how to organize psychophysiological recovery in daily, weekly, monthly classes, as well as throughout the entire annual cycle and how to use digital technologies to process the results obtained in the training process is still not researched.

According to Salier (1936), the general adaptation syndrome, often called stressful, goes through three stages in its development:

1. "reaction to danger" - the body's defenses come to a state of readiness;

2. stage of "resistance" - there is a complete adaptation of the body to stress;

3. stage of "fatigue" - inevitably occurs if the stress is strong enough or its effect is longterm, since the adaptive capacity is limited.

The initial goal of training is to develop physical and mental abilities to genetically possible limits that can only be achieved with regular, scientifically based training. Stress can result from overwork and hypokinesia. Both lead to all sorts of physical deviations and irreparable functional losses. When loads increase in both volume and intensity, the body needs to recover in order to adapt to training stimuli that lead to an increase in the result.

Fatigue is a critical contributor to reduced performance. However, the definition of "fatigue" is a complex problem, both in medicine and in biology. Fatigue after training loads has a characteristic symptomatology, which is expressed in the lack of coordination between the functions of various organs and systems of the body. This contributes to a temporary drop in the level of sports fitness.

From the point of view of psychology, fatigue leads to inhibition at the level of nerve cells, which, in turn, serves as protection against overloads of both the central nervous system and the whole organism.

Fatigue should not be understood only as a negative phenomenon. It becomes a necessary part of the training process and prepares the body for higher loads, meaning adaptation. Fatigue can be local in nature (muscles, cardiovascular system, metabolism, etc.), then subsystems play a major role in the process of its occurrence. With intense sprint loads (for example, in swimming), an impaired acid balance is a factor limiting the growth of athletic performance due to an increase in the concentration of lactate, which occurs as a 
result of anaerobic metabolism, which also develops fatigue. In addition, with prolonged swimming, the energy resources of the muscles are depleted.

Fatigue, therefore, arises as a result of exertion and its effect is not limited to the scope of training and can have effect for a long time after training. During this time, adaptation processes take place in the athlete's body, which are positively influenced by the methods of accelerated psychophysiological recovery. Recovery takes place with the accumulation of energy resources, as a result of which the level of physical fitness increases. If the recovery processes are ineffective and the body's energy reserves are not restored, then fatigue increases and the level of athletic performance decreases.

Repetitive trainings can only be performed after full recovery or using additional recovery aids. In order to continuously increase athletic performance, it is better to work under conditions of incomplete recovery, so that later the supercompensation phase begins. All this is provided in modern training programs, but one should not forget about the degree of recovery. With daily exercise, with appropriate methods, you can achieve either full or partial recovery.

Recovery processes with muscle load can take place before, during and after physical work. It is necessary to pay attention to the following three stages.

First stage. It may seem paradoxical that recovery process takes place before engaging in a training session. However, sports practice, as well as research, indicate that it is impossible to immediately switch to work after rest. There is a stage of operational rest, readiness for upcoming actions, or a warning phase, during which there is an increased accumulation of energy, which is a conditioned reflex process preceding physical activity. This is the stage of motor-vegetative (spinal) reflexes, which depends on the perfection of the reflex itself and the level of energy functioning in relation to the recovery process.

Second stage. Consists in the methodical organization of training directly when performing physical activities. Muscles are in a homeostatic state with an effective swimming technique, when their contraction and relaxation alternate, as well as with the correct organization of training, including rest intervals. Thus, the recovery processes can take place directly during the training work.

Third stage. It is of the greatest importance because it is during this period that most of the adaptation processes can be influenced by a large number of different methods. It is divided into three phases.

In the first phase, there is a transition of functional systems to the baseline state. The duration of the transition is different: both a few seconds and several hours. This is the phase of the not yet complete recovery. Full recovery of all functional systems is carried out due to the fact that training cycles and the sequence in the organization of loads create the prerequisites for avoiding the accumulation of stress.

The second phase is the super compensation phase which, as a rule, is characterized by a "violent" reaction of various functional systems and their adaptation to sports loads.

The third phase is to consolidate the achieved level. Changes under the influence of loads in various functional systems of the body vary widely. The duration of recovery depends on the level of fitness of the athletes. Because early signs of fatigue are difficult to recognize, accelerated recovery is deliberately delayed. Recovery after training and competitive loads can reduce the time required for this by accelerating the transition of functional systems to their basic level.

Accelerated recovery (active and passive processes) is a mandatory factor in the training of highly qualified athletes. As known, the means of accelerated recovery are divided into pedagogical, biomedical and psychological.

Pedagogical means include the optimal training structure, individualization of trainings in accordance with the level of physical fitness, the cyclical nature of the training process the alternation of intense and extensive loads. It is important not forget about the phases of 
active rest. In the final part of the lesson, exercises for active rest and recovery should be performed.

The complex of medico-biological means and methods of recovery consists of a balanced diet, the use of vitamin preparations, physiotherapy (massage, sauna, electrotherapy, hydrotherapy, heliotherapy, movement therapy, etc.) and various pharmacological preparations (ointments, combinations of vitamin and mineralized preparations, glucose, etc.). Medical and biological means create additional opportunities for increasing the level of physical fitness and resistance to specific and nonspecific factors. These factors increase the body's resistance when performing training and competitive loads by reducing the recovery time. Due to this, general and local fatigue is reduced, since energy reserves are restored faster, as well as the elimination of residual products, which has a positive effect on the restoration of the body's natural defenses.

Through the use of mental and psychological means and methods of recovery, mental stress and, consequently, mental fatigue are reduced. These include autogenic training, various methods of relaxation and concentration. The use of any group of means and methods of recovery should be organized in accordance with the level of fitness of athletes. At the same time, it is necessary to exercise objective control over the effectiveness of these methods.

A sign of fatigue is a decrease in the level of sports results when performing standard loads. It is necessary to monitor your workouts through optimal dosing of loads and recovery measures. Means and methods of accelerated recovery are recommended to be used daily during both training and competition. If symptoms of overload become evident, immediate action should be taken. One should not stop training completely (absolute rest). This, as well as stress, can lead to overload.

Successful training of swimmers is impossible without effective control of the athlete's psychophysiological state. Control allows you to determine whether the level of training of an athlete corresponds to the planned value, to reveal the presence of deviations and to find out their nature, to determine the need for corrective actions and to implement them. To successfully integrate technological knowledge into the training context, coaches must have sufficient knowledge of information technology. These skills are critical to the training process and must be integrated with pedagogical and meaningful knowledge. The coach must know not only the training and psychophysiological process that he plans for athletes, but also how the training process can be revised after analyzing the use of digital technologies.

The development and application of the system of automated planning of the training process, proposed in Fig. 1, will allow by the computational and analytical way to quickly construct models of various options for conducting training, compare them with each other and select the most preferable among them. This overcomes the limited sampling of empirical material with which coaches who plan a real educational and training process deal with. 
environmental conditions

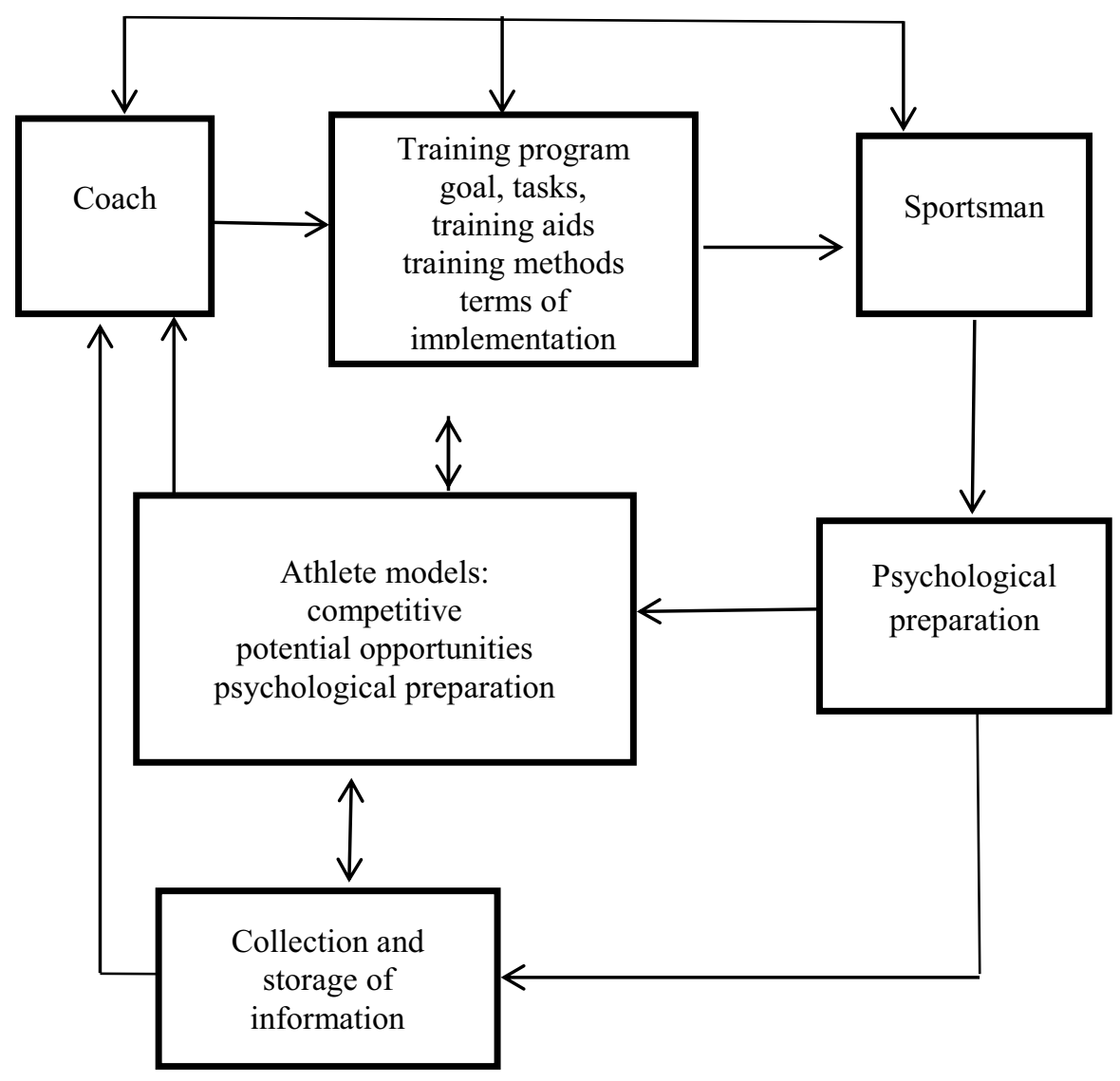

Fig.1. The composition of the system for the automated planning of the training process.

\section{Discussion}

The relationship between technology, training process and content is vital, and the coaching staff can revise this structure in order to quickly make decisions after collecting and analyzing information about the athlete's condition, thanks to the introduction of digital technologies into the practice of the training process and psychological adaptation. Digital technology cannot replace the coach's job, but it will help improve and innovate the training process. The use of digital technology presents a good opportunity to obtain more objective and complex data in real time with devices that are more informational, accurate and easier to use.

In addition, it is necessary to take into account the daily frequency of the athlete's mental state. The rational distribution of loads at each stage of the training process is just as important as during the long-term, perspective training of athletes. The success of solving this problem depends on the qualifications of the trainer, as well as on further research.

Information technology and the ability to structure them help to compile the mass of necessary documentation and methods for transferring information from athlete to coach and vice versa. At the moment, the main problem is the lack of tools and programs in the 
sports environment for operational information processing, which are so necessary for their formation and application in the training process.

The next most important factor is the need for a coaching staff who owns not only professional competencies, but also knows how to work with Internet resources in modern educational programs. The methodology for preparing the coaching staff to work with the use of information technology is not included in the training courses for advanced training. At the same time, the need for personnel possessing information technologies is increasing more and more. In our country, such education does not meet the real needs of the rapidly developing digitalization of sports education.

\section{Conclusions}

The key direction in the development of modern digital technologies in the training process is the construction of the training process in close connection between athletes and the coach, informative transmission of the athlete's psychophysiological state during and after training, data collection and processing by means of information technologies. This will make it possible to correct the training process in the best way and improve the results of athletes, which was confirmed by our experiment.

In the course of a pedagogical experiment aimed at studying the influence of connections between physical work and psychophysiological recovery, used after training and competitive loads, as well as the need to use digital technologies to ensure the effectiveness of the training process, the following indicators were obtained in the experimental and control groups. The data show that the increase in speed among athletes in the experimental group is more significant than in the testing group. This is shown in table. 1, which shows the values of the increase in the swimming speed (v) and the reduction in the time of swimming the distance $(t)$ in absolute and relative values. The analysis of the data in the table shows that the result of swimming a distance of $50 \mathrm{~m}$ by the athletes of the control group improved by an average of 0.17 seconds, while the effectiveness of the athletes of the experimental group improved by 0.29 seconds. The effectiveness of athletes in the control group swimming the distance of $100 \mathrm{~m}$ improved by $1.07 \mathrm{sec} .(1.8 \%)$, and the experimental one - by $2.16 \mathrm{sec}$. $(3.7 \%)$.

The development and application of digital technologies will make it possible to computationally and analytically construct models of various training options, compare them and select preferred ones, thereby overcoming, to a certain extent, the limited samples of empirical material that trainers who plan a real educational and training process are dealing with, taking into account psychophysiological characteristics of an athlete. Psychophysiological recovery is part of the training process. Information technologies make it possible to more mobilely receive information about the psychophysiological changes in an athlete in the training process. Recovery procedures should be included in training programs and planned both within a single day, as well as a week or a month.

The effect of training largely depends on the correct balance of stress and psychophysiological recovery. It is necessary to use various methods in order to ensure an accelerated recovery from stress.

\section{References}

1. A.S. Solodkov, Scientific-theoretical journal "Scientific Notes of the University named after Lesgaft P.F." 6 (100), 131-143 (2013).

2. E.V. Bolshova, Features of the criteria for assessing the functional state of swimmers using discriminant analysis, Educational-patriotic and physical culture and sports 
activities in universities: solving urgent problems: materials of the international scientific and practical conference (April 19, 2019), ed. Khromina S. I. Tyumen: Tyumen Industrial University, 40-45, (2019).

3. Yu.V. Koryagina, Fundamental research 10 (8), 1753-1757 (2013).

4. AJ Coutts, Int J Sports Physiol Perform 9, 741 (2014).

5. M. Cardinale, M.C. Varley, Int J Sports Physiol Perform 12, 1-23, (2016).

6. M Korner, S Butof, C Muller, L Zimmermann, Becker S, and J Bengel, J Interprof Care 30, 15-28 (2016).

7. X. Schelling, L.Torres-Ronda, Strength Cond J., 72-80 (2016).

8. M. André, Digital technology in physical education: global perspectives (London, Routledge, 2018).

9. A. Casey, V.A. Goodyear, K.M. Armour, Digital technologies and learning in physical education: Pedagogical cases (Routledge, London, 2016)

10. A. Casey, V.A. Goodyear, K.A. Armour, Sport, Education and Society 22, 288-304 (2017).

11. T. Hopper, K. Sanford, H. Fu, Digital technology in physical education: global perspectives (London, Routledge, 2018).

12. M. Kok, J. van der Kamp, Digital technology in physical education: global perspectives (London, Routledge, 2018).

13. D. Lupton, Sport, Education and Society 20 (1), 122-132 (2015).

14. D. Memmert, Almond Bunker, D. Butler, J. Fasold, F. Griffin, L. Nopp, Research Quarterly for Exercise and Sport 86(4), 347-359 (2015).

15. I.D. Graevskaya, JI.A. Ioffe, Theory and practice of physical culture - M., FiS 4, 32-36 (1973). 